\title{
Efficient photon coupling from a diamond nitrogen vacancy center by integration with silica fiber
}

\author{
Rishi N Patel ${ }^{1,2}$, Tim Schröder ${ }^{1}$, Noel Wan ${ }^{1}$, Luozhou Li ${ }^{1}$, Sara L Mouradian ${ }^{1}$, Edward H Chen $^{1}$ \\ and Dirk R Englund ${ }^{1}$
}

A central goal in quantum information science is to efficiently interface photons with single optical modes for quantum networking and distributed quantum computing. Here, we introduce and experimentally demonstrate a compact and efficient method for the low-loss coupling of a solid-state qubit, the nitrogen vacancy (NV) center in diamond, with a single-mode optical fiber. In this approach, single-mode tapered diamond waveguides containing exactly one high quality NV memory are selected and integrated on tapered silica fibers. Numerical optimization of an adiabatic coupler indicates that near-unity-efficiency photon transfer is possible between the two modes. Experimentally, we find an overall collection efficiency between $16 \%$ and $37 \%$ and estimate a single photon count rate at saturation above $700 \mathrm{kHz}$. This integrated system enables robust, alignment-free, and efficient interfacing of single-mode optical fibers with single photon emitters and quantum memories in solids.

Light: Science \& Applications (2016) 5, e16032; doi:10.1038/Isa.2016.32; published online 12 February 2016

Keywords: diamond nanophotonics; fiber optics; nitrogen vacancy center; quantum optics; single photon source

\section{INTRODUCTION}

Efficient coupling of stationary quantum memories to a single spatial mode in silica fiber is of central importance in a range of quantum information processing applications, including long-distance entanglement of stationary qubits and quantum networks ${ }^{1-5}$. Recently, the efficient fiber coupling of atomic quantum memories also enabled large atom-cavity coupling ${ }^{6-8}$ and strong single-atom nonlinearities ${ }^{9,10}$. Among solid-state qubits, the nitrogen vacancy (NV) center in diamond has emerged as an attractive quantum memory due to its optically addressable and long coherence electronic and nuclear spin states $^{11,12}$. These properties have enabled recent demonstrations of heralded quantum entanglement ${ }^{13}$ and teleportation ${ }^{14}$ between two separated NV centers. To improve the entanglement probability in such schemes, an open experimental challenge is to improve the efficiency with which single photons from an NV center can be channeled into a single-guided optical mode. This has motivated a variety of light collection approaches, including diamond micro-posts ${ }^{15}$, solid immersion lenses ${ }^{16-18}$, grating structures ${ }^{19,20}$, and inverse tapered coupling to photonic integrated circuits ${ }^{21}$. Several research efforts have also sought to integrate quantum emitters directly with optical fibers, as a way of eliminating non-essential optical components and achieving compact and nearly monolithic interfaces. Recently, fluorescence collection from colloidal quantum dots and diamond nanocrystals containing single NVs was proposed ${ }^{22,23}$ and demonstrated, often using a tapered fiber section ${ }^{24-30}$. However, these approaches use point-like emitters that exhibit poor collection efficiency; in addition, the spin and optical properties of NVs in diamond nanocrystals are degraded compared to bulk diamond ${ }^{23}$. It is desirable instead to transfer photons from emitters in a wave-guiding structure to an optical fiber, as was recently demonstrated for quantum dots ${ }^{31}$. Here we introduce an approach that uses adiabatic power transfer between a tapered silica fiber and a single-mode diamond micro-waveguide fabricated from high-quality CVD-grown diamond. This integrated diamondsilica waveguide system enables efficient optical collection from highquality NV centers.

\section{MATERIALS AND METHODS}

Device description

We consider a tapered single-mode diamond micro-waveguide in direct contact with the tapered section of a single-mode optical fiber, as illustrated in Figure 1. The diamond micro-waveguide is positioned by van der Waals forces directly on the tapered fiber resulting in coupling between the optical modes of the two structures.

For a slowly varying diamond width, corresponding to a slowly varying effective refractive index, light in the diamond micro-waveguide remains in the fundamental mode of the combined diamondsilica structure. We analyze this problem using coupled mode analysis, following a similar analysis for coupling between a silicon waveguide to a tapered fiber ${ }^{32}$. Figure 2 a plots the effective indices of the diamond and fiber modes as a function of the diamond waveguide cross-section, for a fixed fiber diameter of $500 \mathrm{~nm}$. Near a diamond cross-section of $140 \mathrm{~nm}$, a clear anti-crossing is observed as the matching group indices of the waveguides results in strong mode-coupling between them. The corresponding mode fields are shown in Figure $2 \mathrm{~b}$. If the diamond

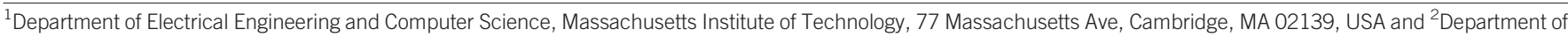
Applied Physics, Stanford University, 348 Via Pueblo Mall, Stanford, CA 94305, USA 


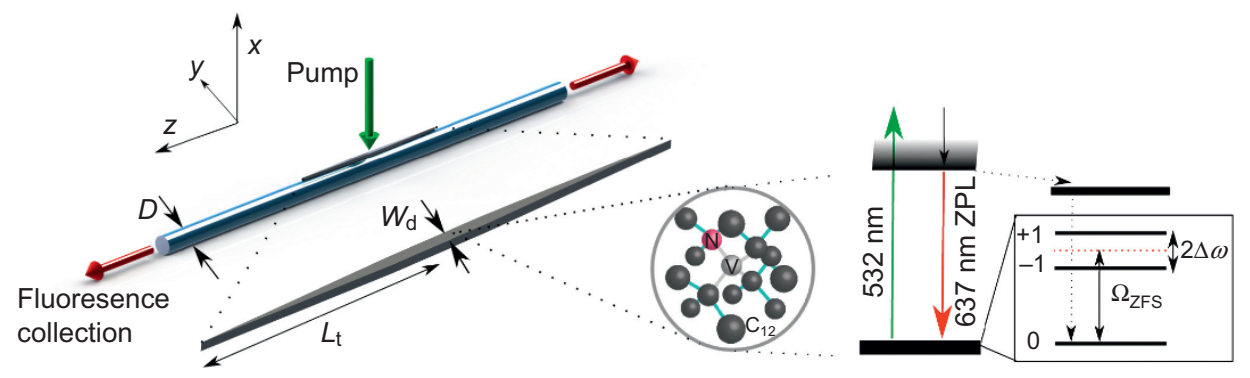

Figure 1 System overview. A diamond micro-waveguide containing an NV center (gray) in parallel contact with a tapered fiber segment (blue) is schematically shown. Excitation and collection pathways are shown in green and red, respectively. The inset shows the diamond crystal lattice and the NV center spectrum. The parameters in this experiment are the fiber diameter $D \approx 500 \mathrm{~nm}$, the diamond width (at $z=0$ ), $W_{\mathrm{d}}(0)=200 \mathrm{~nm}$, and the taper length $L_{t}=5 \mu \mathrm{m}$. In the NV energy level diagram, the zero-phonon line is denoted by ZPL, the zero-field-splitting frequency of $2.87 \mathrm{GHz}$ by $\Omega_{\text {ZFS }}$ and the Zeeman splitting between the +1 and -1 states in a direct current (DC) magnetic field by $2 \Delta \omega$.

taper is swept slowly such that the optical mode remains in the ground state through the transition from the diamond into the silica waveguide, nearly $100 \%$ power transfer is possible. Specifically, the diamond taper width $W_{d}(z)$ must satisfy:

$$
\frac{d W_{\mathrm{d}}(z)}{d z} \ll\left(\frac{d n_{\mathrm{d}}}{d W_{\mathrm{d}}(z)}\right)^{-1} \frac{2 \pi}{\lambda_{0}}\left(\Delta n_{\mathrm{eff}}\right)^{2}
$$

where $\frac{d n_{\mathrm{d}}}{d W_{d}(z)}$, the rate of change of the fundamental diamond mode effective index versus diamond width, can be computed from the derivative of the uncoupled diamond band plot shown in Figure 2a. The parameters on the right-hand side of Equation (1) can be estimated from the simulation shown in Figure 2a. Using $\lambda_{0}=637 \mathrm{~nm}$,
$\Delta n_{\mathrm{eff}}=0.13$, and $\frac{d n_{d}}{d W_{d}(z)}=0.008$, we thus obtained a bound $\frac{d W_{d}(z)}{d z}<0.02$. For $W_{d}(0)=200 \mathrm{~nm}$, this corresponds to a taper length $L_{t}=10 \mu \mathrm{m}$. This is consistent with the finite-difference timedomain (FDTD) calculation shown in Figure 2c, confirming that the coupling efficiency increases with the length of the diamond waveguide, as expected.

Next, we consider how the orientation of the NV, which is assumed to be located at the center of the diamond micro-waveguide (see Figure 1), affects the coupling efficiency. In our simulation, we consider three orthogonal dipole polarizations along the axes shown in the inset of Figure 1a. For an $x$-polarized dipole, a theoretical overall

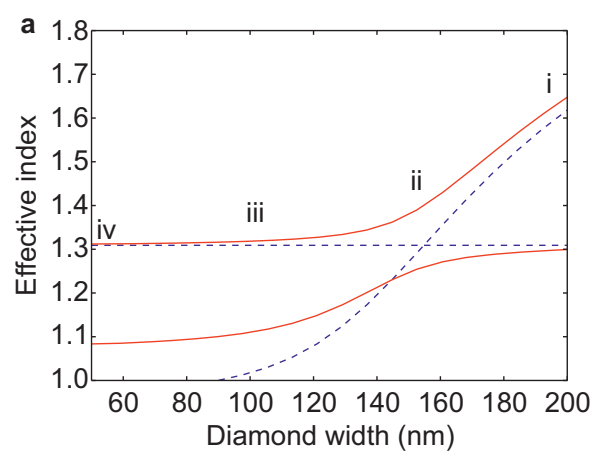

b
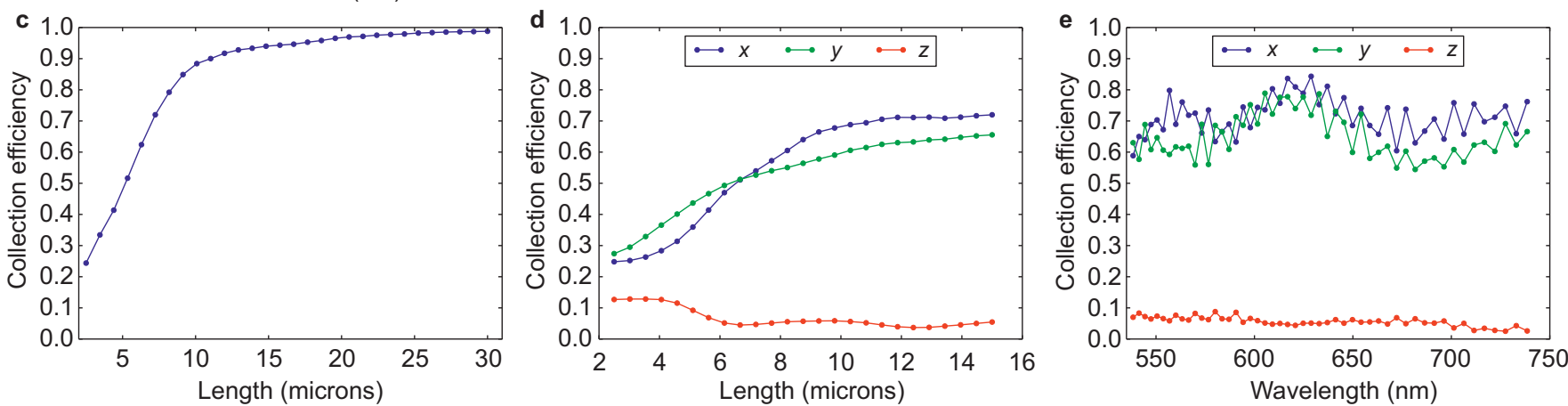

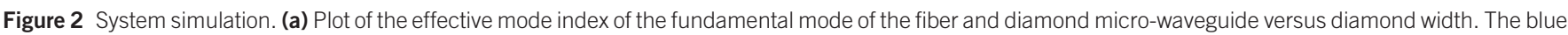

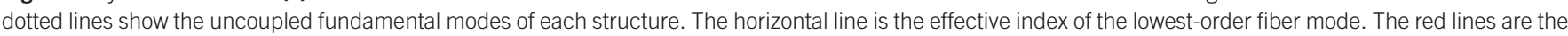

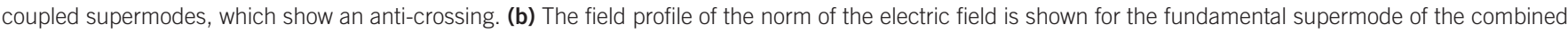

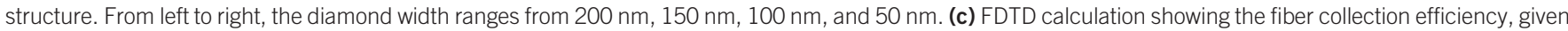
that only the fundamental supermode of the diamond-fiber structure is excited. (d) FDTD calculation using a dipole source of three different polarizations. Blue, green,

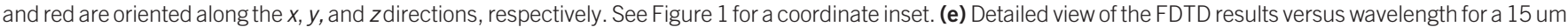
long diamond micro-waveguide. 
collection efficiency of over $70 \%$ can be achieved, as can be seen from the collection efficiency plot for the three dipole orientations in Figure $2 \mathrm{~d}$. We also note that the adiabatic coupling approach has the advantage of being highly broadband, as is apparent from the FDTD calculations plotted versus excitation wavelength (Figure 2e).

\section{Device fabrication}

The system fabrication involves three main stages. First, tapered fibers were fabricated from single-mode optical fibers (Thorlabs 630HP) using a standard heat-and-pull technique ${ }^{33}$ while secured in a tapered fiber mount that is sealed to maintain a clean-air environment and to block air flow. Our setup uses a fixed hydrogen flame and two motorized stages. To prepare the fiber for tapering, we strip a $1 \mathrm{~cm}$ region of the outer jacket and preheat the region for $120 \mathrm{~s}$. We then pull the stages in opposite directions at a speed of $30 \mu \mathrm{m} \mathrm{s}^{-1}$. Laser transmission at $630 \mathrm{~nm}$ is monitored throughout the pulling process, and the stages are stopped manually when the transmission begins to fall. We confirmed with scanning electron micrscope and optical images that this diameter is on the order of $500 \mathrm{~nm}$.

The fiber tapering results in an adiabatic fiber mode conversion to maintain a high $(\approx 90 \%)$ transmission of $633 \mathrm{~nm}$ laser light. Although especially long tapers can provide efficiencies around $97 \%{ }^{34}$, we used here shorter tapers as they are easier to manipulate. Second, diamond micro-waveguides were fabricated from a $200 \mathrm{~nm}$ thin film of $\left[\begin{array}{lll}1 & 0 & 0\end{array}\right]$ electronic grade synthetic diamond using transferred hard mask lithography ${ }^{35}$. They are $12 \mu \mathrm{m}$ long, with a $5 \mu \mathrm{m}$ triangular taper on either side of a $2 \mu \mathrm{m}$ section of constant width (Figure $3 \mathrm{a}$ ). Third, we characterized and selected individual diamond micro-waveguides containing exactly one NV center before placing them onto the waist of the tapered fiber, where they adhere readily by van der Waals forces. This detachment and placement is done using a tungsten micromanipulator tip (Ted Pella). We use a rotation stage for the fiber to align it parallel to the micro-waveguide, while the tungsten probe is controlled by a separate three-axis piezo stage. Figure $3 \mathrm{~b}$ and $3 \mathrm{c}$ shows a completed device under a scanning electron microscope and optical confocal microscope, respectively.

\section{Confocal excitation and autocorrelation measurements}

A home-built confocal microscope is used to excite the NV center. We use a $532 \mathrm{~nm}$ pump laser and focus it onto our sample using a Zeiss $\mathrm{NA}=0.75$ cover-glass corrected objective. We couple the signal from each fiber end to free-space in order to filter out the pump with two $550 \mathrm{~nm}$ long-pass filters. The resulting signal is coupled back into
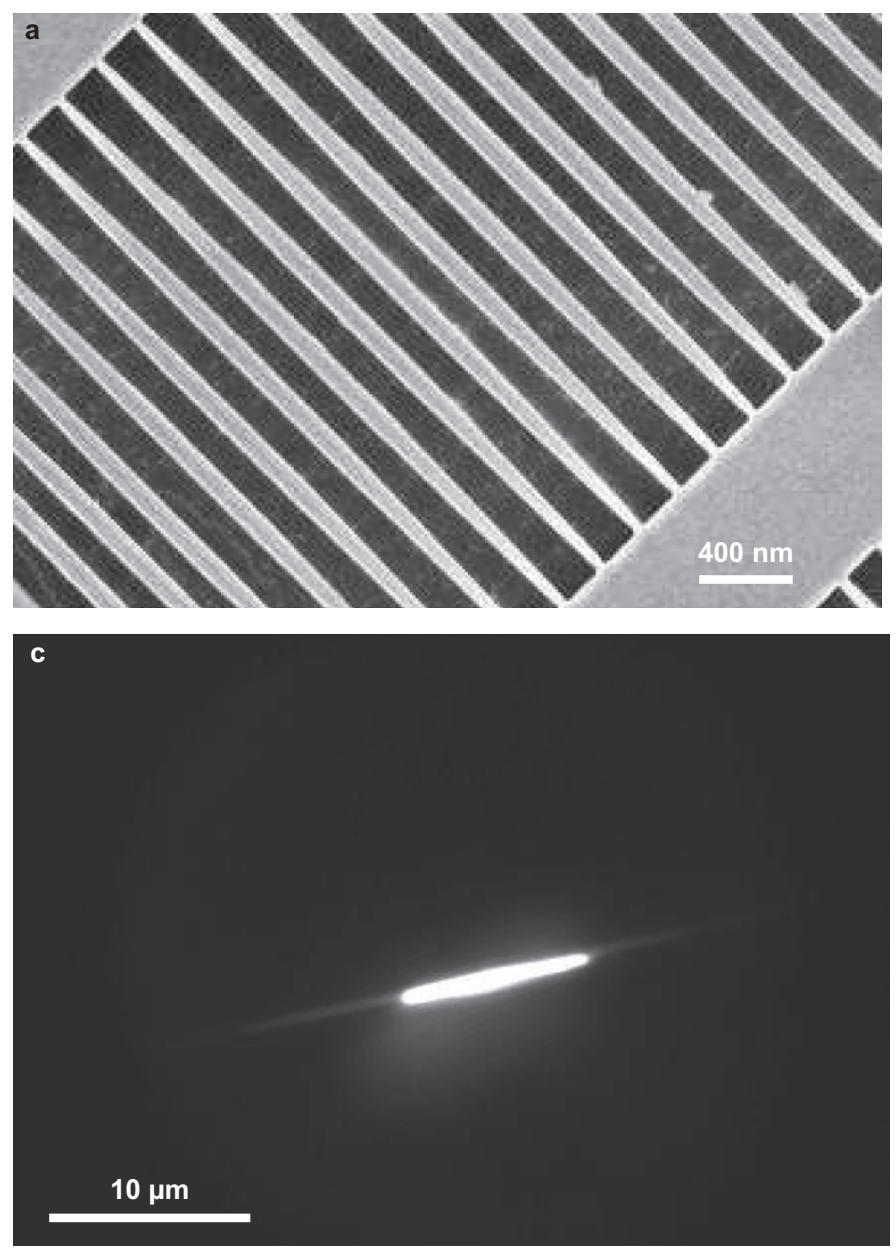
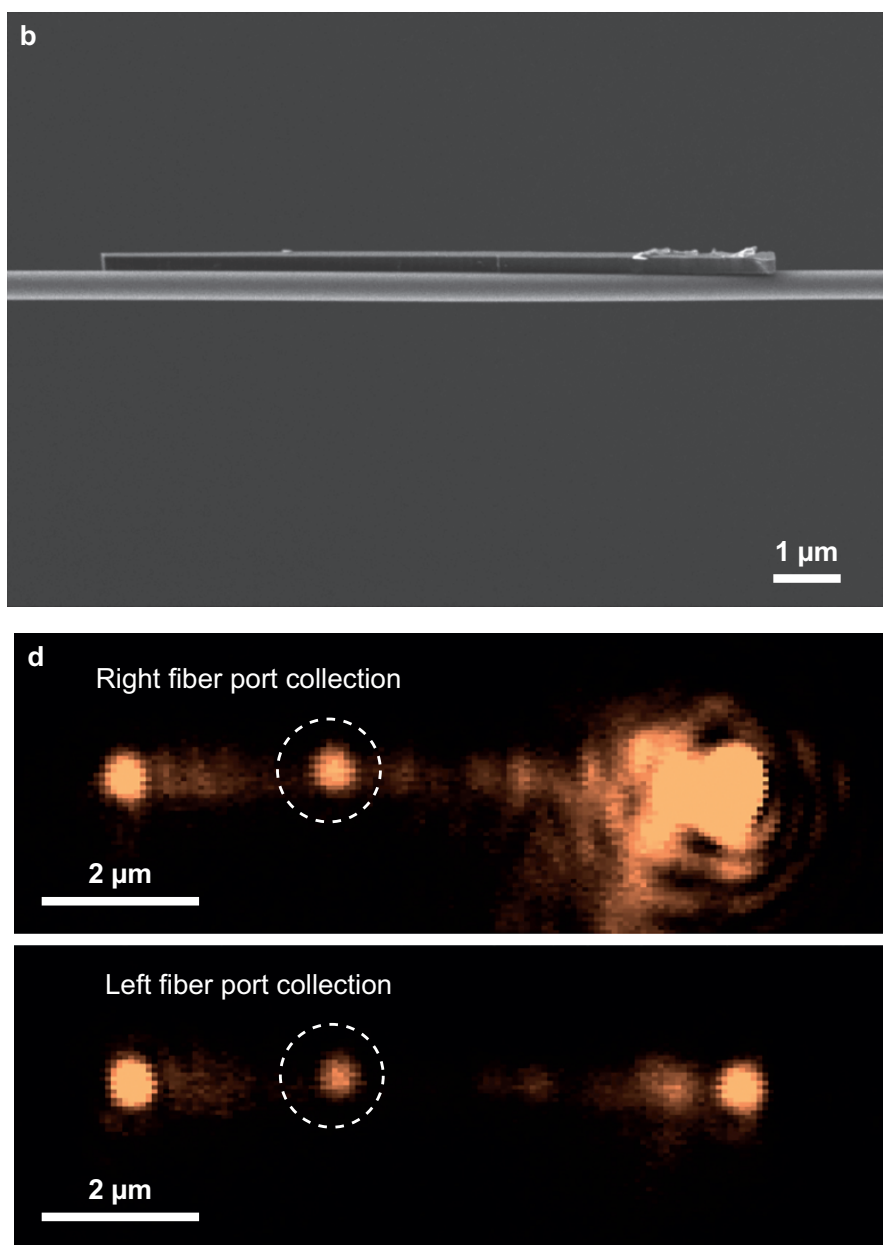

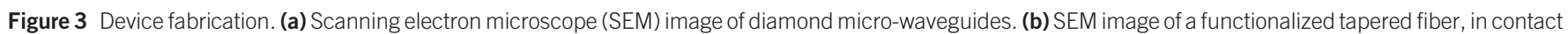

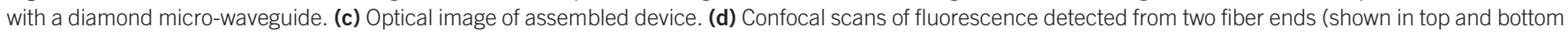

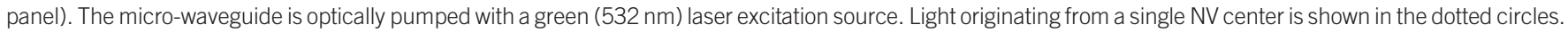


single-mode (SMF-28, Corning) fiber before being directed to two avalanche photo-diodes (APDs; Perkin-Elmer). We perform crosscorrelation measurements with a histogram of start-stop time intervals using a counting module with a bin width of $0.256 \mathrm{~ns}$. In a confocal scan, the NV can be identified as a bright fluorescence spot to the left of the center of the micro-waveguide (Figure $3 \mathrm{~d}$ ). Here, we excited the NV from the top while collecting photons through the left and right fiber ends, as seen in the top and bottom panels, respectively. The ends of the diamond micro-waveguides are also apparent as bright fluorescence spots on the scan. At these positions, more laser power is scattered into the fiber, causing increased fluorescence background. The bright extended spot at the right of the top panel is due to additional asymmetric scattering from the tip of the diamond waveguide. This scattering signal does not originate from NV center fluorescence, as determined from the photon statistics and spectra from these points (see Supplementary Fig. S4).

\section{RESULTS AND DISCUSSION}

\section{Optical characterization}

Optical measurements reveal the efficient collection of NV fluorescence directly coupled into a single-guided mode. We detected NV fluorescence through both the left and right fiber ends, as evidenced by the typical NV spectrum with a pronounced zero phonon line around $637 \mathrm{~nm}$ (Figure 4a).

By varying the pump power, we observe a saturation behavior in the $\mathrm{NV}$ signal (Figure $4 \mathrm{~b}$ and $4 \mathrm{c}$ ). All fits are to a model of the form:

$$
I(P)=\frac{P I_{\text {sat }}}{P_{\text {sat }}+P}+C P
$$

where $I$ is the measured intensity in counts per second (cps), $P$ is the pump power, $I_{\text {sat }}$ the saturation intensity, $P_{\text {sat }}$ the saturation pump power, and $C$ is a fitting parameter that characterizes the linear background contribution. The fits estimate an overall $I_{\text {sat }}$ of $(712 \pm 24) \cdot 10^{3}$ cps from both fiber ends combined versus $(16 \pm 2) \cdot 10^{3}$ cps from the objective. The corresponding saturation powers are approximately 380 and $485 \mu \mathrm{W}$, respectively. We measured the normalized second-order autocorrelation function of the fiber-coupled light with a Hanbury Brown-Twiss setup, using the fiber itself as the intrinsic beamsplitter. At an incident pump power of $114 \mu \mathrm{W}$, the anti-bunching with $g^{(2)}(0)=(0.15 \pm 0.02)<0.5$ indicates the presence of a single NV center; no background was subtracted from this measurement (Figure 4d). This autocorrelation data is fit to a three-level model:

$$
g^{(2)}(\tau)=1+p_{f}^{2}\left(c e^{-|\tau| / \tau_{1}}-(1+c) e^{-|\tau| / \tau_{2}}\right)
$$

where $p_{f}$ is the single photon emission probability, $\tau_{1}$ is a time constant for the central anti-bunching dip, $\tau_{2}$ is the time constant for correlation function decay at intermediate times, and $c$ is a parameter describing the amplitude of the bunching shoulders. We then estimated the background by plotting the parameter $g^{(2)}(0)=1-p_{f}^{2}$ for different values of the excitation pump power (Figure 4e). Here we identify a maximum incident pump power of $2.25 \mathrm{~mW}$ focused to a
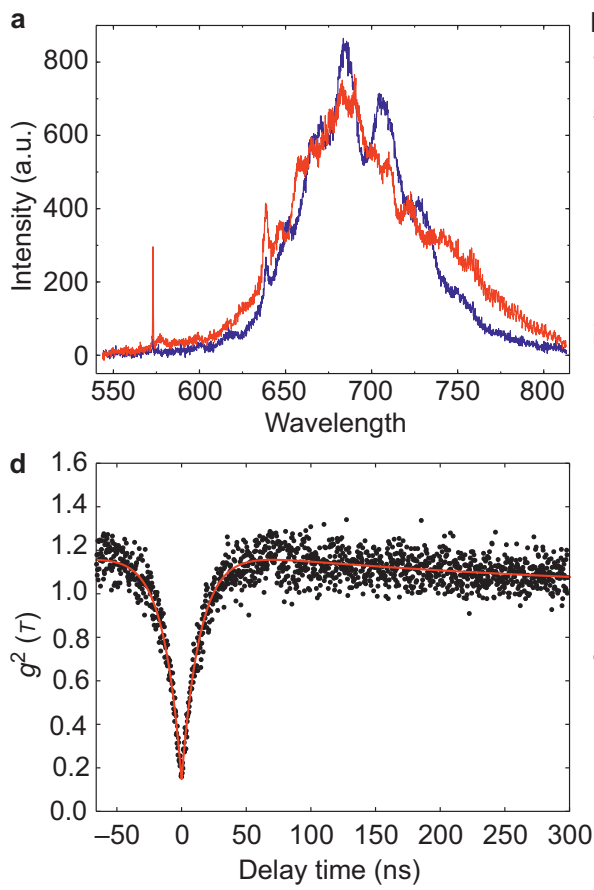
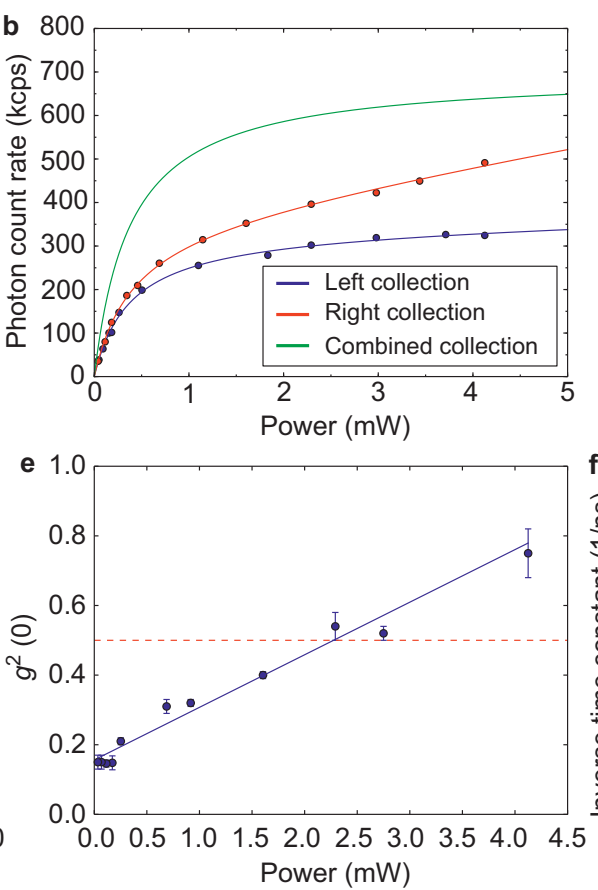
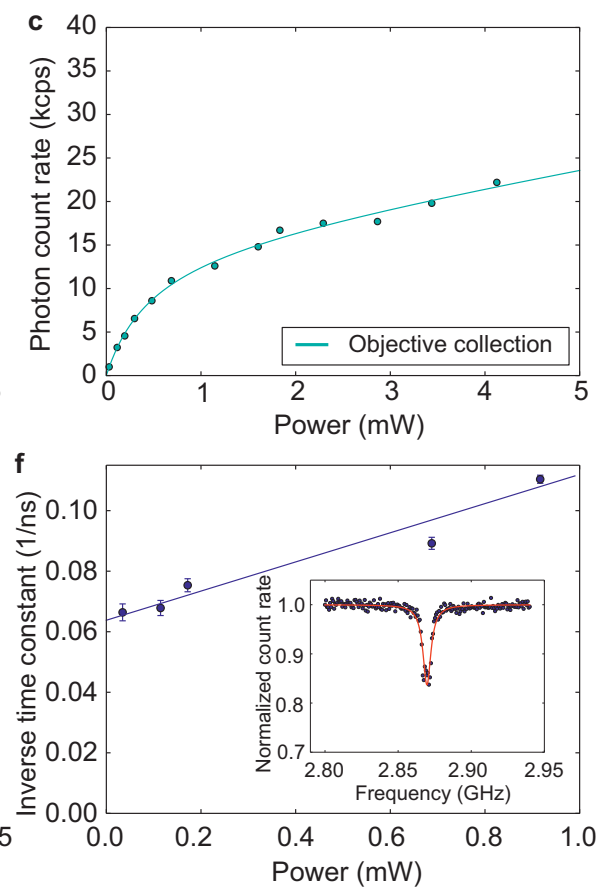

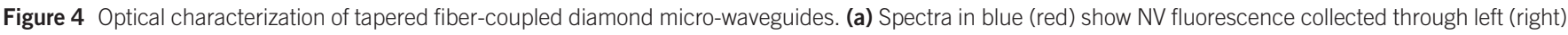

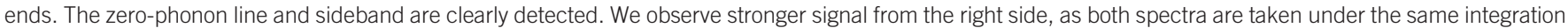

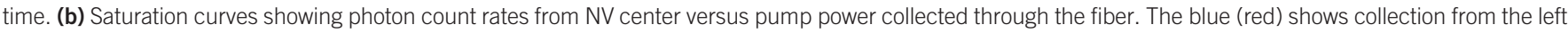

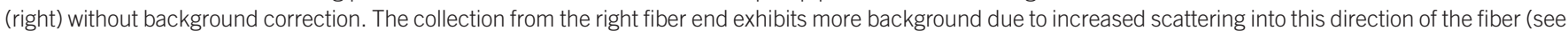

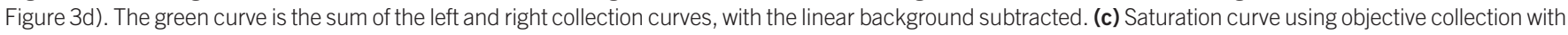

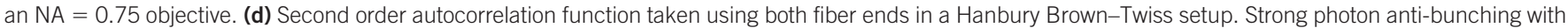

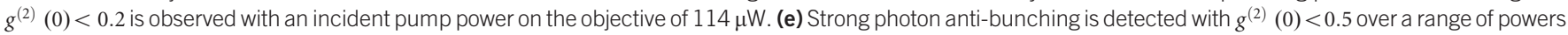

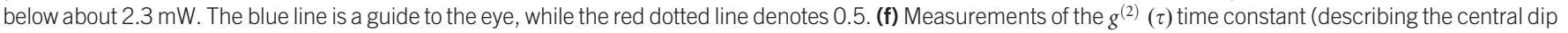

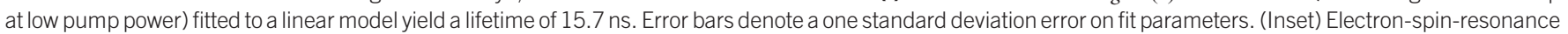
fluorescence dip in zero external DC magnetic field. 
spot size of approximately $0.6 \mu \mathrm{m}$, beyond which the background emission causes $g^{(2)}(0)>0.5$. At this pump power, we use the green curve in Figure $4 \mathrm{~b}$ to show that over 600000 single photons per second are detected. Fitting Equation (3) for a number of different pump powers, allows us to extract the inverse time constant, $1 / \tau_{1}$ (Figure $\left.4 \mathrm{f}\right)^{15}$. We see that $1 / \tau_{1}$ varies linearly for small pump power and extrapolation to zero power yields a lifetime, $\tau_{\mathrm{NV}}$, of $15.7 \pm 1.1 \mathrm{~ns}$.

\section{System efficiency}

We use two different metrics to characterize our observed system efficiency. The first, relating the number of guided photons to the total possible number of photons emitted with a unity quantum efficiency, is given by:

$$
\beta=\frac{\Gamma_{\text {guided }}}{\Gamma_{\text {decay }}}
$$

where $\Gamma_{\text {guided }}$ is the total excited state decay rate into the guided modes (into the fiber), and $\Gamma_{\text {decay }}$ is the total decay rate of the NV center (including both non-radiative and radiative pathways). $\beta$ is a lower bound on the observed system efficiency if one assumes an NV quantum efficiency of unity. We estimate the parameter $\Gamma_{\text {guided }}$ from our measurements of the observed count rate:

$$
\Gamma_{\text {guided }}^{*}=\eta_{\text {apd }} \kappa_{\text {tg }} \kappa_{\text {taper }} \Gamma_{\text {guided }}
$$

where $\eta_{\text {apd }}$ is the quantum efficiency of our APD detectors, $\kappa_{\text {taper }}$ the transmission efficiency of our tapered fiber, and $\kappa_{\text {tg }}$ the transmission efficiency of our filtering stages. In our experiment, $\eta_{\text {apd }} \approx 65 \%$, $\kappa_{\text {taper }} \approx 95 \%$, and $\kappa_{\mathrm{tg}} \approx 43 \%$. $\kappa_{\text {taper }}$ is obtained from transmission measurements of the fiber during fabrication, and represents an upper bound on the taper transmission. It is defined as the single-sided transmission, from the center of the taper to either of the fiber ends. $\kappa_{\mathrm{tg}}$ is obtained from measuring the transmission of a $635 \mathrm{~nm}$ laser signal through both left and right filtering ports, providing an upper bound for the filter stage transmission. We take a weighted average of these two measurements for our estimated $\kappa_{\mathrm{tg}}$ and $\Gamma_{\text {guided }}^{*}=(712 \pm 24) \cdot 10^{3}$ cps directly from the parameter $I_{\text {sat }}$ above. Dividing out the transmission factors gives a total single photon count rate of about $\Gamma_{\text {guided }}=(2.7 \pm 0.1) \cdot 10^{6} \mathrm{cps}$. We determine $\Gamma_{\text {decay }}$ as the inverse of the lifetime, $15.7 \pm 1.1 \mathrm{~ns}$. Finally, this gives a value of $\beta \approx 4.2 \%$. This lower bound of $\beta$ does not take into account the NV's charge instability or the non-unity quantum efficiency.

In a second method of estimating the collection efficiency, we use the ratio of the guided photons to the total number of radiated photons:

$$
\eta_{\mathrm{c}}=\frac{1}{1+\frac{\Gamma_{\text {free }}}{\Gamma_{\text {guided }}}}
$$

where $\Gamma_{\text {free }}$ is the decay rate into free space modes. $\eta_{\mathrm{c}}$ differs from the first metric because it requires an estimation of the total number of radiated photons as opposed to the total decay rate, which includes both non-radiative and radiative terms. That is, we can write $\eta_{c}=\beta / \eta_{\mathrm{QE}}$, where $\eta_{\mathrm{QE}}$ is the quantum efficiency of the NV. We estimate the free space decay rate from:

$$
\Gamma_{\text {free }}^{*}=\eta_{\text {apd }} \kappa_{\text {tf }} \kappa_{\mathrm{NA}} \Gamma_{\text {free }}
$$

where $\kappa_{\mathrm{tf}}$ is the transmission efficiency of our confocal microscope setup and $\kappa_{\mathrm{NA}}$ gives the fraction of photons collected into the acceptance angle of our microscope objective (NA $=0.75$, cover glass-corrected Zeiss). This fraction requires an estimate of the $\mathrm{NV}$ orientation, which in our case can be obtained because we work with a $\left[\begin{array}{lll}1 & 0 & 0\end{array}\right]$ oriented diamond thin film and the facet of this sample, to a good approximation, faces the objective (See Supplementary Information for additional details) ${ }^{36}$. With FDTD simulations, we then obtained $\kappa_{\mathrm{NA}}$. We also estimated a range for $\kappa_{\mathrm{tf}}$ based on transmission measurements of our confocal setup, and taking into account losses from the cover glass enclosing our sample. From saturation measurements acquired via the objective, we have $\Gamma_{\text {free }}^{*}=(16 \pm 2) \cdot 10^{3}$ cps. Using the factors $\kappa_{\mathrm{tf}}^{\text {low }} \approx 1 \%, \kappa_{\mathrm{tf}}^{\text {high }} \approx 3 \%$, and $\kappa_{\mathrm{NA}} \approx 18 \%$, we estimate an efficiency of $\eta_{\mathrm{c}}^{\text {lower }} \approx 16.4 \%$ and $\eta_{\mathrm{c}}^{\text {upper }} \approx 37.0 \%$. The large range of $\kappa_{\mathrm{tf}}$ is caused by our collection optics, as the fiber coupling efficiency is strongly dependent on the position of the sealing cover slip in the focus, and the setting of the correction ring of the objective. By comparison, our FDTD-based calculation yields a lower and upper bound on the collection efficiency of 0.36 and 0.61 , respectively. We attribute the discrepancy primarily to the assumption in our simulation of a triangularly shaped diamond micro-waveguide tapered with ends going to zero width, an assumption that is not valid in the experimental system. In addition, the NV is not located exactly in the center of the adiabatic coupler, and may have different coupling efficiencies to either side. In practice, we can achieve micro-waveguide tips of about $50 \mathrm{~nm}$ which creates a sudden index step at which scattering losses can occur. Using the above estimates for the collection efficiency, we obtain quantum efficiency bounds of $\eta_{\mathrm{NV}}^{\text {lower }} \approx 11.3 \%$ and $\eta_{\mathrm{NV}}^{\text {upper }} \approx 25.6 \%$.

The inset of Figure $4 \mathrm{f}$ shows continuous wave optically detected magnetic resonance of our NV electron spin. Separate Hahn-echo measurements on waveguides produced in the same way indicate long phase coherence times in excess of $100 \mu$ s (see Supplementary Fig. S2) ${ }^{37}$.

\section{Discussion}

For our system to be useful in larger photonic networks, multiple working devices must be fabricated. In our study, we assembled four devices similar to the one discussed in detail. In three of these systems, we measured anti-bunching with $g^{(2)}(0)<0.5$, and in one $g^{(2)}(0) \approx 0.5$ indicating the presence of a single emitter and two closely spaced emitters, respectively. The count rates for these devices were on the order of several hundred thousand counts, similar to the device discussed above. In addition, we note that the yield of the diamond waveguides we fabricated was between $5 \%$ and $10 \%$. This is defined as the fraction of waveguides that contain a single NV near the center.

It is important to cover several limitations of our system, and to highlight some aspects for future work. We observed that the tapered fiber transmission degrades over time because of the deposition of large scatterers, such as dust. In future experiments, this can be averted by mounting the tapered section in a sealed container. Furthermore, presently our method is limited by diamond fabrication capabilities to make slowly tapering structures, as we currently attach the waveguides on the ends to a diamond substrate. With improved fabrication techniques, diamond waveguides could be made with longer taper lengths and thinner tips, improving adiabatic power transfer up to the theoretical limit close to unity. Regarding our optical pumping scheme, we currently use confocal excitation to excite individual NV centers in our devices. For a completely fiber-integrated approach, it would be interesting to use a fiber-integrated Bragg filter to allow excitation from one fiber end and collection through the second fiber end. Such a system could be cooled by simple immersion in a cryogenic fluid, eliminating the need for a confocal microscope and in principle allowing for lifetime-limited ZPL emission, an important requirement for a number of quantum information experiments. 


\section{CONCLUSION}

We have demonstrated an approach for the efficient integration of a high-quality quantum memory directly with a silica fiber. With a raw single photon detection rate exceeding $6 \cdot 10^{5} \mathrm{cps}$ and $g^{(2)}(0)<0.5$, we show a roughly fourfold improvement over previous fiber-coupled approaches that used diamond point emitters instead of wave-guiding structures ${ }^{16}$. In addition, the fiber background is strongly suppressed allowing exceptionally low $g^{(2)}(0) \approx 0.15$ (without background subtraction) for cross-correlation measurements in a fiber-integrated system. This geometry can be used for coherent spin manipulation of a fiber-coupled spin qubit, thereby providing efficient optical access to a long-lived quantum memory. The deterministic pick and place method presented here is amenable to larger scale integration and can be extended to the evanescent integration of diamond-based nano-cavities ${ }^{38}$. Furthermore, a transmission configuration could open up possibilities for long-distance quantum communication experiments with completely fiber-integrated components.

\section{ACKNOWLEDGEMENTS}

R.N.P. thanks Amir H. Safavi-Naeini for helpful discussion and Christopher Foy for useful comments on the manuscript. R.N.P. was supported in part by the MIT SuperUROP (Undergraduate Research Opportunities Program). T.S. was supported by the Alexander von Humboldt-Foundation. E.H.C. was supported by the NASA Office of the Chief Technologist's Space Technology Research Fellowship. Experiments were carried out in part with support from the Air Force Office of Scientific Research PECASE (supervised by G. Pomrenke). S.L.M. was supported in part by the AFOSR Quantum Memories MURI and by a fellowship from the NSF iQuISE program, award number 0801525. Fabrication of the diamond micro-waveguides was carried out in part at the Center for Functional Nanomaterials, Brookhaven National Laboratory, which is supported by the US Department of Energy, Office of Basic Energy Sciences, under Contract No. DE-AC02-98CH10886.

1 Kimble HJ. The quantum internet. Nature 2008; 453: 1023-1030.

2 Northup TE, Blatt R. Quantum information transfer using photons. Nat Photonics 2014; 8: 356-363.

3 Childress L, Hanson R. Diamond NV centers for quantum computing and quantum networks. MRS Bull 2013; 38: 134-138.

4 Lončar M, Faraon A. Quantum photonic networks in diamond. MRS Bull 2013; 38 : 144-148.

5 Sprague MR, Michelberger PS, Champion TFM, England DG, Nunn J et al. Broadband single-photon-level memory in a hollow-core photonic crystal fibre. Nat Photonics 2014; 8: 287-291.

6 Thompson JD, Tiecke TG, de Leon NP, Feist J, Akimov AV et al. Coupling a single trapped atom to a nanoscale optical cavity. Science 2013; 340: 1202-1205.

7 Tiecke TG, Thompson JD, de Leon NP, Liu LR, Vuletić V et al. Nanophotonic quantum phase switch with a single atom. Nature 2014; 508: 241-244.

8 Tiecke TG, Nayak KP, Thompson JD, Peyronel T, de Leon NP et al. Efficient fiberoptical interface for nanophotonic devices. Optica 2015; 2: 70-75.

9 O'Shea D, Junge C, Volz J, Rauschenbeutel A. Fiber-optical switch controlled by a single atom. Phys Rev Lett 2013; 111: 193601.

10 Mitsch R, Sayrin C, Albrecht B, Schneeweiss P, Rauschenbeutel A. Directional nanophotonic atom-waveguide interface based on spin-orbit interaction of light. arXiv: 1406.0896. 2014.

11 Maurer PC, Kucsko G, Latta C, Jiang L, Yao NY et al. Room-temperature quantum bit memory exceeding one second. Science 2012; 336: 1283-1286.

12 Bar-Gill N, Pham LM, Jarmola A, Budker D, Walsworth RL. Solid-state electronic spin coherence time approaching one second. Nat Commun 2013; 4: 1743.

13 Bernien H, Hensen B, Pfaff W, Koolstra G, Blok MS et al. Heralded entanglement between solid-state qubits separated by 3 meters. Nature 2013; 497: 86-90.
14 Pfaff W, Hensen BJ, Bernien H, van Dam SB, Blok MS et al. Unconditional quantum teleportation between distant solid-state quantum bits. Science 2014; 345: 532-535.

15 Babinec TM, Hausmann BJM, Khan M, Zhang YN, Maze JR et al. A diamond nanowire single-photon source. Nat Nanotechnol 2010; 5: 195-199.

16 Schröder T, Gädeke F, Banholzer MJ, Benson O. Ultrabright and efficient singlephoton generation based on nitrogen-vacancy centres in nanodiamonds on a solid immersion lens. New J Phys 2011; 13: 055017.

17 Hadden JP, Harrison JP, Stanley-Clarke AC, Marseglia L, Ho YLD et al. Strongly enhanced photon collection from diamond defect centers under microfabricated integrated solid immersion lenses. Appl Phys Lett 2010; 97: 241901.

18 Siyushev P, Kaiser F, Jacques V, Gerhardt I, Bischof S et al. Monolithic diamond optics for single photon detection. Appl Phys Lett 2010; 97: 241902.

19 Choy JT, Bulu I, Hausmann BJM, Janitz E, Huang IC et al. Spontaneous emission and collection efficiency enhancement of single emitters in diamond via plasmonic cavities and gratings. App/ Phys Lett 2013; 103: 161101.

20 Li LZ, Chen EH, Zheng JB, Mouradian SL, Dolde F et al. Efficient photon collection from a nitrogen vacancy center in a circular bullseye grating. Nano Lett 2015; 15 : 1493-1497.

21 Hausmann BJM, Shields B, Quan QM, Maletinsky P, McCutcheon M et al. Integrated diamond networks for quantum nanophotonics. Nano Lett 2012; 12: 1578-1582.

22 Chonan S, Kato S, Aoki T. Efficient single-mode photon-coupling device utilizing a nanofiber tip. Sci Rep 2014; 4: 4785.

23 Almokhtar M, Fujiwara M, Takashima H, Takeuchi S. Numerical simulations of nanodiamond nitrogen-vacancy centers coupled with tapered optical fibers as hybrid quantum nanophotonic devices. Opt Express 2014; 22: 20045-20059.

24 Yalla R, Le Kien F, Morinaga M, Hakuta K. Efficient channeling of fluorescence photons from single quantum dots into guided modes of optical nanofiber. Phys Rev Lett 2012; 109: 063602.

25 Schröder T, Schell AW, Kewes G, Aichele T, Benson O. Fiber-integrated diamondbased single photon source. Nano Lett 2011; 11: 198-202.

26 Schröder T, Fujiwara M, Noda T, Zhao HQ, Benson $\mathrm{O}$ et al. A nanodiamond-tapered fiber system with high single-mode coupling efficiency. Opt Express 2012; 20: 10490-10497.

27 Liebermeister L, Petersen F, Münchow AV, Burchardt D, Hermelbracht J et al. Tapered fiber coupling of single photons emitted by a deterministically positioned single nitrogen vacancy center. Appl Phys Lett 2014; 104: 031101.

28 Liu XD, Cui JM, Sun FW, Song XR, Feng FP et al. Fiber-integrated diamond-based magnetometer. Appl Phys Lett 2013; 103: 143105.

29 Fujiwara M, Toubaru K, Noda T, Zhao HQ, Takeuchi S. Highly efficient coupling of photons from nanoemitters into single-mode optical fibers. Nano Lett 2011; 11: 4362-4365

30 Henderson MR, Gibson BC, Ebendorff-Heidepriem H, Kuan K, Afshar V et al. Diamond in tellurite glass: a new medium for quantum information. Adv Mat2011; 23: 28062810.

31 Davanço M, Rakher MT, Wegscheider W, Schuh D, Badolato A et al. Efficient quantum dot single photon extraction into an optical fiber using a nanophotonic directional coupler. App/ Phys Lett 2011; 99: 121101.

32 Gröblacher S, Hill JT, Safavi-Naeini AH, Chan J, Painter O. Highly efficient coupling from an optical fiber to a nanoscale silicon optomechanical cavity. Appl Phys Lett 2013; 103: 181104.

33 Hauer BD, Kim PH, Doolin C, MacDonald AJR, Ramp H et al. On-chip cavity optomechanical coupling. EPJ Tech Instrum 2014; 1: 1-21.

34 Vetsch E, Reitz D, Sagué G, Schmidt R, Dawkins ST et al. Optical interface created by laser-cooled atoms trapped in the evanescent field surrounding an optical nanofiber. Phys Rev Lett 2010; 104: 203603.

35 Li LZ, Bayn I, Lu M, Nam CY, Schröder T et al. Nanofabrication on unconventional substrates using transferred hard masks. Sci Rep 2015; 5: 7802.

36 Alegre TPM, Santori C, Medeiros-Ribeiro G, Beausoleil RG. Polarization-selective excitation of nitrogen vacancy centers in diamond. Phys Rev B 2007; 76: 165205.

37 Mouradian SL, Schröder T, Poitras CB, Li LZ, Goldstein J et al. Scalable integration of long-lived quantum memories into a photonic circuit. Phys Rev X2015; 5: 031009.

38 Li LZ, Schröder T, Chen EH, Walsh M, Bayn I et al. Coherent spin control of a nanocavity-enhanced qubit in diamond. Nat Commun 2015; 6: 6173.

\footnotetext{
(c) (i) (2) This license allows readers to copy, distribute and transmit the Contribution as long as it attributed back to the author. Readers are permitted to alter, transform or build upon the Contribution as long as the resulting work is then distributed under this is a similar license. Readers are notpermitted touse the Contribution for commercial purposes. Please read the full license for further details at http://creativecommons.org/licenses/ by-nc-sa/4.0/
} 